\title{
Effet de la gestion des feuilles d'élagage du palmier à huile sur le stock de carbone et les propriétés physico-chimiques du sol dans les palmeraies villageoises du Bénin
}

\author{
Hervé Nonwègnon Sayimi Aholoukpè (1), Guillaume Lucien Amadji ${ }^{(2)}$, Didier Blavet ${ }^{(3)}$, \\ Jean-Luc Chotte ${ }^{(3)}$, Philippe Deleporte ${ }^{(4)}$, Bernard Dubos ${ }^{(5)}$, Albert Flori ${ }^{(5)}$, \\ Christophe Jourdan ${ }^{(4)}$ \\ (1) Centre de Recherches agricoles Plantes Pérennes. INRAB. BP 01 Pobè (Bénin). E-mail : aholoukpeherve@yahoo.fr \\ (2) Université d'Abomey-Calavi. Faculté des Sciences agronomiques. BP 526. Cotonou (Bénin). \\ (3) IRD. UMR Éco\&Sols. 2, Place Viala. F-34060 Montpellier Cedex 2 (France). \\ (4) CIRAD. UMR Éco\&Sols. 2, Place Viala. F-34060 Montpellier Cedex 2 (France). \\ (5) CIRAD. UPR Systèmes de Pérennes. Avenue Agropolis. F-34398 Montpellier Cedex 5 (France).
}

Reçu le 29 avril 2015, accepté le 12 novembre 2015.

Description du sujet. Au Bénin, la culture du palmier à huile est largement répandue en milieu villageois. L'étude porte sur l'impact du recyclage des feuilles d'élagage sur les propriétés du sol des palmeraies.

Objectifs. Évaluer l'effet de deux modes de gestion des feuilles élaguées dans la palmeraie sur le stock de carbone et les propriétés physico-chimiques du sol.

Méthode. Six palmeraies villageoises de deux classes d'âges (7-12 ans et 13-24 ans) ont été retenues dans le sud-est du Bénin afin de comparer les propriétés du sol de deux zones : les zones de restitution totale des feuilles d'élagage (RT) et celles de restitution nulle des feuilles $(\mathrm{RN})$ représentant le témoin. Dans chaque palmeraie, le sol sous chaque modalité de recyclage des feuilles a été prélevé, sur fosses, jusqu'à $50 \mathrm{~cm}$ de profondeur à proximité de six palmiers. Les propriétés du sol ont été déterminées par des méthodes d'analyse conventionnelles et par la spectroscopie proche infrarouge.

Résultats. Les stocks de carbone et d'azote sous RT à l'âge adulte sont de $58 \mathrm{Mg} \cdot \mathrm{ha}^{-1}$ et $4,3 \mathrm{Mg} \cdot \mathrm{ha}^{-1}$, respectivement et sont significativement supérieurs aux stocks dans tous les autres traitements $\left(34,6 \mathrm{Mg} \mathrm{C} \cdot \mathrm{ha}^{-1}\right.$ et $\left.2,9 \mathrm{Mg} \mathrm{N} \cdot \mathrm{ha}^{-1}\right)$. Le recyclage des feuilles a amélioré la fertilité du sol jusqu'à $20 \mathrm{~cm}$ de profondeur dans les palmeraies adultes. La porosité et la densité apparente du sol ont été aussi améliorées.

Conclusions. La gestion des feuilles élaguées dans les palmeraies modifie les propriétés du sol qui sont nettement améliorées en surface après 10 ans de recyclage des feuilles.

Mots-clés. Elaeis guineensis, pratique culturale, élimination des feuilles, propriétés physico-chimiques du sol, carbone, azote, fertilité du sol, Bénin.

Effect of management of pruned fronds of oil palm on soil carbon stock and soil physico-chemical properties in Beninese smallholder plantations

Description of the subject. In Benin, oil palm plantations are widely managed by smallholder farmers. The study addresses the impact on soil properties of the recycling of pruned fronds from these trees.

Objectives. The study aims to assess the effect of two modes of management of pruned fronds on the carbon stock and physico-chemical properties of soil.

Method. Six oil palm plantations were selected to represent two age classes (7-12 years and 13-24 years) in southeastern Benin. They were used to compare the soil properties of two areas: the areas of total recycling of pruned fronds (TR) and the areas of no recycling of the fronds (NR), which represented the control. In each plantation, soil samples were collected under the two treatments, from holes of up to $50 \mathrm{~cm}$ depth, near six oil palm trees. The soil properties were determined using conventional laboratory methods and near-infrared spectroscopy method.

Results. Carbon and nitrogen stocks under TR of adult plantations were $58 \mathrm{Mg} \cdot \mathrm{ha}^{-1}$ and $4.3 \mathrm{Mg} \cdot \mathrm{ha}^{-1}$, respectively, and were significantly higher than the stocks in all other treatments (34.6 Mg C.ha- ${ }^{-1}$ and $2.9 \mathrm{Mg} \mathrm{N} \cdot h \mathrm{~h}^{-1}$ ). Recycling of pruned fronds 
improved soil fertility at a depth of up to $20 \mathrm{~cm}$ in the adult plantations. The porosity and bulk density of the soil were also improved.

Conclusions. The management of the pruned fronds in the smallholders' oil palm plantations modified the soil properties, which were significantly improved in the top soil after 10 years of frond recycling.

Keywords. Elaeis guineensis, cultural practices, defoliation, physico-chemical properties of soil, carbon, nitrogen, soil fertility, Benin.

\section{INTRODUCTION}

Le palmier à huile (Elaeis guineensis) cultivé sous les latitudes tropicales et subtropicales humides, est l'oléagineux ayant le rendement en corps gras le plus élevé (Barcelos, 1998 ; Corley et al., 2003). Originaire d'Afrique, le palmier à huile a connu une forte expansion dans le monde, en particulier en Asie du Sud-Est, où la Malaisie et l'Indonésie détiennent aujourd'hui plus de $80 \%$ des superficies mondiales plantées (Ahoyo, 2008). Ce succès asiatique s'explique par des conditions climatiques globalement plus favorables que celles de son aire d'origine (Nodichao, 2008). En Afrique de l'Ouest, où il est cultivé depuis la fin du $19^{\mathrm{e}}$ siècle, les conditions climatiques sont parfois marginales et limitent fortement les rendements, comme c'est le cas au Bénin. Dans ce pays où la culture n'est maintenant pratiquée que par des villageois, la station de recherche sur le palmier à huile située à Pobè a développé un programme spécifique de mise au point de matériel végétal adapté à la sècheresse (Nouy et al., 1999). Parallèlement, les programmes de recherche ont étudié l'amélioration de la productivité par une fertilisation minérale adaptée, la mise au point de techniques culturales et la gestion de la matière organique du sol dans le but de réduire l'impact des déficits hydriques (Djègui et al., 1992 ; Adjè, 2004).

Dans une palmeraie, les principales sources de matière organique qui peuvent être recyclées tout au long du cycle de culture sont constituées des rafles après égrappage des régimes, des sous-produits d'usinage (boues et effluents) et des feuilles d'élagage. La biomasse des troncs des palmiers ne peut être recyclée qu'au moment de la replantation. Tous ces produits contribuent à augmenter le stock de matière organique du sol et à améliorer les propriétés physiques et chimiques du sol, ce qui a un effet positif sur le rendement du palmier (Khalid et al., 2000a; Khalid et al., 2000b ; Corley et al., 2003). Parmi les éléments nutritifs restitués par le recyclage des feuilles, l'azote et le potassium sont deux éléments majeurs en raison des quantités libérées dans le sol et en raison de leur importance pour la nutrition minérale du palmier à huile (Khalid et al., 1999 ; Dubos et al., 2011).

Dans les palmeraies villageoises du Bénin en phase productive, les biomasses qui sont recyclées directement au champ sont les résidus des cultures vivrières associées aux jeunes palmiers jusqu'à 5 ans, puis les feuilles d'élagage des palmiers (Djègui et al., 1992; Aholoukpè et al., 2013). Ces feuilles représentent en moyenne $8 \mathrm{t}$ et $4 \mathrm{t}$ de biomasse sèche par hectare par an respectivement dans une palmeraie adulte et pré-adulte (Aholoukpè, 2013). Elles peuvent être :

- soit disposées en tas sur le sol où elles se décomposeront; elles forment des andains discontinus situés à mi-distance sur la ligne séparant deux palmiers consécutifs (Figure 1) ;

- soit exportées hors de la parcelle pour un usage domestique (combustible de cuisine, palissade, etc.).

C'est ce dernier usage qui est le plus fréquent (Aholoukpè et al.,2013). Si l'exportation de la biomasse du palmier à huile a un effet négatif sur le maintien de la matière organique et sur la fertilité du sol (Khalid et al., 2000a), très peu d'études ont toutefois évalué le potentiel de la pratique de recyclage des résidus de récolte du palmier à huile dans l'amélioration des stocks organiques et la restitution des nutriments du sol (Djègui, 1992 ; Law et al., 2009 ; Nelson et al., 2011).

Dans une première étude sur la station de recherche de Pobè, Djègui (1995) a travaillé sur la variabilité spatiale de la matière organique du sol liée d'une part aux pratiques culturales propres aux cultures vivrières conduites sur billons dans les jeunes plantations et, d'autre part, au mode de plantation en ligne avec restitution en andains des feuilles d'élagage. Ces essais conduits en station n'ont cependant pas pris en compte l'effet sur le long terme de la restitution des feuilles d'élagage dans les conditions des palmeraies villageoises où la pratique de fertilisation disparait au moment de la mise en production. L'objectif général de l'étude présentée dans cet article est d'évaluer l'effet du recyclage ou non de la biomasse des feuilles sur les stocks de matière organique et la fertilité du sol en milieu réel, en étudiant des palmeraies villageoises soumises pendant des durées plus ou moins longues aux différentes pratiques. De façon spécifique, deux questions de recherche ont été étudiées :

- effet du mode de gestion des feuilles élaguées et de l'âge des palmeraies sur les stocks de carbone et d'azote du sol ;

- effet du mode de gestion des feuilles élaguées sur les propriétés physico-chimiques des sols des palmeraies adultes. 
a

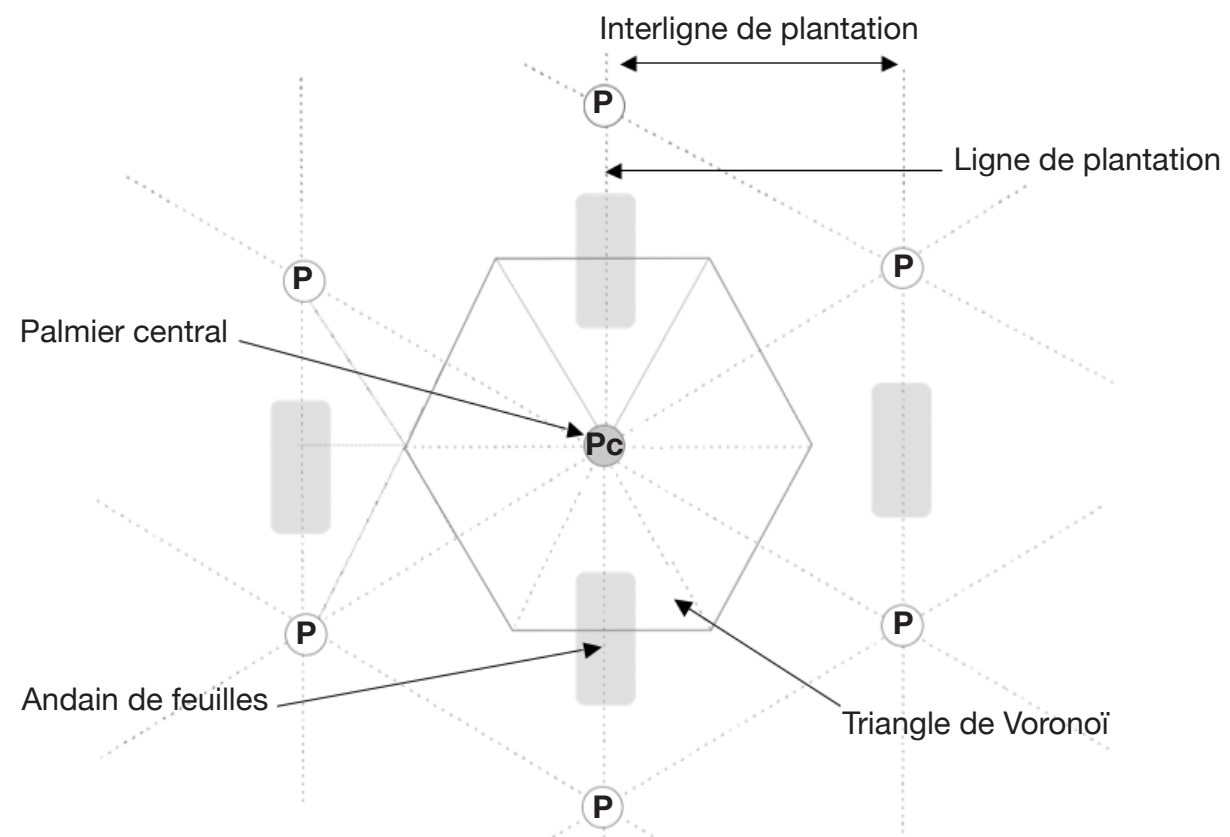

b

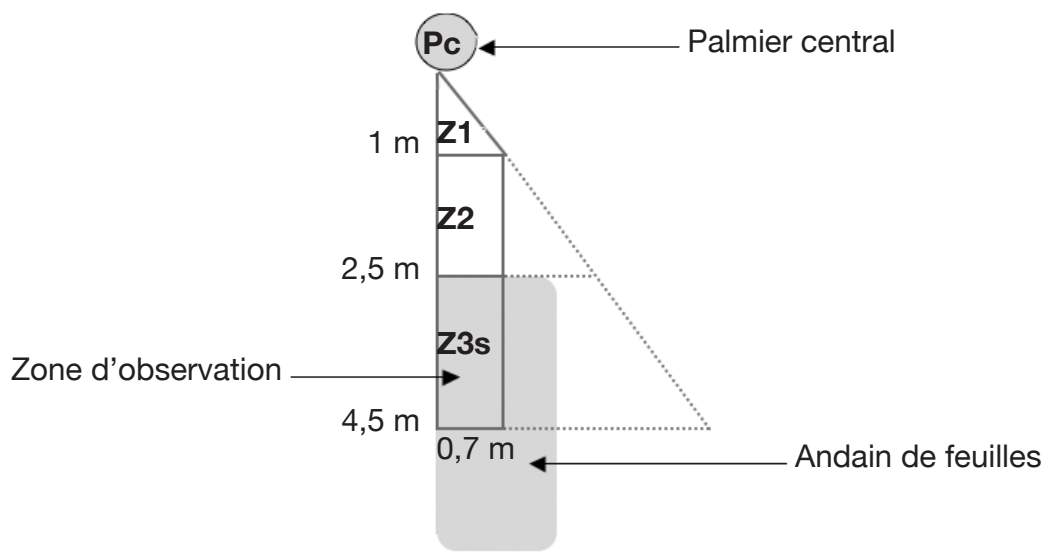

Figure 1. Espace de Voronoï autour du palmier étudié (a) et triangle de Voronoï (b) présentant les dimensions de la zone Z3s de prélèvement du sol sous andain et dans l'interligne - Complete Voronoï space around the studied palm tree (a) and Voronoï triangle $(\boldsymbol{b})$ with the dimensions of Z3s zone for the soil sampling under the pruned fronds pile and inside the interrow.

a. L'espace de Voronoï est l'hexagone défini par six palmiers qui entourent le palmier central étudié. Il est constitué de 12 triangles rectangles égaux (Nodichao, 2008). Pour les six palmiers choisis par palmeraie, il a été installé six espaces de Voronoï dont le palmier central (Pc) est chaque fois utilisé pour les observations - The Voronoï space is the hexagon delimited by six palm trees which surround the studied palm tree. This space is split into 12 equivalent right triangles (Nodichao, 2008). For the six palm trees choseen by palm plantation, six Voronoï spaces have been set up and the central tree $(P c)$ was each time used for the observations; $\mathbf{b}$. Le triangle de Voronoï est composé de 5 zones dont les zones Z1, Z2 et Z3s délimitent l'espace simplifié (Dassou, 2011). Les observations ont été faites dans la zone 3 simplifiée (Z3s) - The Voronoï triangle includes 5 zones of which Z1, Z2 and Z3s delimite the simplified space (Dassou, 2011). The observations were done inside the simplified zone 3 (Z3s).

\section{MATÉRIEL ET MÉTHODES}

\subsection{Zone d'étude}

L'étude a été conduite dans le département du Plateau situé au sud-est du Bénin $\left(2^{\circ} 30-2^{\circ} 45 \mathrm{E}\right.$ et $6^{\circ} 35$ - $\left.7^{\circ} 45 \mathrm{~N}\right)$. Le climat est de type subéquatorial, caractérisé par une alternance de deux saisons pluvieuses (avril à juillet et mi-septembre à mi-novembre) et deux saisons sèches (aout à mi-septembre et mi-novembre à mars). Dans la zone d'étude, on enregistre une hauteur moyenne de $1300 \mathrm{~mm}$ de pluie par an et un 
déficit hydrique ${ }^{1}$ du sol estimé entre 600 et $800 \mathrm{~mm}$, ce qui limite la productivité du palmier à huile (Nouy et al., 1999). Les sols étudiés sont largement représentés dans le département et sont les plus cultivés avec le palmier à huile (Aholoukpè et al., 2013). Ils sont communément appelés « terre de barre » et ont été décrits par Azontondé (1991) et Amadji et al. (2008). Selon la nomenclature française, ce sont les sols ferrallitiques faiblement désaturés appauvris en argile (Volkoff et al., 1976). Dans la classification de la FAO, il s'agit de ferralsols rhodiques, molliques sous forêt (teneur en $\mathrm{C}>0,6 \%$ ) et ochriques (teneur en $\mathrm{C}<0,6 \%$ ) sous culture (Djègui et al., 1992).

Dans la zone d'étude, la végétation est dominée par des savanes arborées et arbustives, des palmeraies plantées avec du matériel végétal sélectionné («palmeraies sélectionnées ») et des palmeraies issues de régénération naturelle («palmeraies naturelles »), des jachères arbustives, des îlots de forêts semi-décidues et des galeries forestières (Djègui, 1992).

\subsection{Sélection des palmeraies}

Les deux communes produisant le plus de palmiers à huile dans le département (Adja-Ouèrè et Sakété) (Adjadi, 2012 ; Akpo et al., 2012) ont été retenues pour le choix de six palmeraies villageoises distantes entre elles d'un minimum de $10 \mathrm{~km}$ et dont quelques caractéristiques sont présentées dans le tableau 1. S'agissant d'une étude en milieu villageois, il n'a pas été possible d'identifier des palmeraies d'âges identiques à l'échelle du département. De ce fait, les palmeraies étudiées ont été regroupées en classes d'âges définies dans une étude typologique préalable (Aholoukpè et al., 2013). Les classes des palmeraies pré-adultes (7-12 ans) et adultes (13-24 ans) ont été retenues pour l'étude. Elles ont été plantées selon le même dispositif en triangles équilatéraux de $9 \mathrm{~m}$ de coté (143 palmiers par hectare) et ont été conduites selon des itinéraires techniques similaires: précédents culturaux constitués d'anciennes cultures annuelles, association de cultures vivrières au jeune âge, très rares apports d'engrais minéraux à partir de 5 ans, élagage à partir de 7 ans et dépôt en andains des feuilles d'élagage. Dans ces palmeraies, on a distingué les zones où sont déposées toutes les feuilles d'élagage entre deux palmiers sur la ligne de plantation (modalité RT, restitution totale des feuilles) et les zones non concernées par le recyclage dans les interlignes de plantation (modalité RN, restitution nulle). Les andains de feuilles couvrent une surface assimilée à un rectangle centré sur la ligne séparant deux palmiers consécutifs et dont la superficie moyenne est

\footnotetext{
${ }^{1}$ Une estimation du déficit hydrique est obtenue à partir du bilan simplifié basé sur la pluviosité mensuelle, une réserve hydrique du sol fixée à $150 \mathrm{~mm}$ et une ETP de 100 à $150 \mathrm{~mm}$ par mois selon la saison (Nouy et al., 1999).
}

d'environ $7 \mathrm{~m}^{2}$. Ils représentent $10 \%$ de la superficie d'une palmeraie (Aholoukpè, 2013). La modalité RN est assimilable à la pratique d'exportation totale des feuilles d'élagage rencontrée dans les palmeraies villageoises de la zone d'étude (Aholoukpè et al., 2013). Les classes d'âges correspondent respectivement à une moyenne de 3 et 10 ans de recyclage des feuilles élaguées.

L'étude comprend deux phases :

- dans un premier temps, les six palmeraies ont servi à étudier les stocks de carbone et d'azote du sol sur 0-50 $\mathrm{cm}$ de profondeur. Le dispositif a permis d'étudier l'effet des deux facteurs « mode de gestion des feuilles d'élagage » $(\mathrm{RT}$ ou $\mathrm{RN})$ et « classe d'âge » (pré-adulte : 7-12 ans ou adulte : 13-24 ans) et leur interaction ;

- dans un second temps, les conclusions de la première phase de l'étude nous ont amenés à compléter les observations des trois palmeraies de la classe d'âge adulte par des analyses physico-chimiques $(\mathrm{pH}$, cations échangeables, capacité d'échange cationique, densité apparente et porosité du sol) en distinguant les modalités RT et RN.

\section{3. Échantillonnage de sol et mesures de terrain}

Dans chaque palmeraie étudiée, les observations ont été faites pour six palmiers tirés de façon aléatoire sur une superficie de 1 ha. La surface plantée a été structurée selon un dispositif de Voronoï (Figure 1) qui définit des secteurs triangulaires équivalents autour du palmier (Nodichao, 2008). Pour comparer les modalités RT et $\mathrm{RN}$, nous avons restreint les zones d'études aux rectangles $\mathrm{Z} 3 \mathrm{~s}$ du dispositif de Voronoï simplifié proposé par Dassou (2011). Des fosses de dimensions 1,80 m x $0,70 \mathrm{~m} \times 1 \mathrm{~m}$ (longueur, largeur et profondeur) ont été creusées dans chacune des zones (andains et interligne) des six palmiers de la parcelle. Les analyses physicochimiques et la détermination de la densité apparente du sol ont été faites sur les couches de profondeurs 0-5, $5-10,10-20,20-30$ et $30-50 \mathrm{~cm}$. Aucun profil de sol n'a donc été pris en compte dans cet échantillonnage. Pour chaque fosse, les quatre faces ont été utilisées pour prélever les échantillons de sol qui ont servi à constituer des échantillons composites pour les analyses chimiques de chaque fosse. Au total, 360 échantillons composites de sol ont été réalisés sur la base des cinq profondeurs de prélèvement, des deux fosses par palmier, des six palmiers par palmeraie et des six palmeraies retenues pour l'étude. Chaque face de la fosse a aussi été utilisée pour déterminer la densité apparente moyenne du sol au moyen d'un cylindre de $3 \mathrm{~cm}$ de diamètre et de $5 \mathrm{~cm}$ de hauteur.

\section{4. Étude de laboratoire}

Les sols ont été séchés à l'air et tamisés à $2 \mathrm{~mm}$ pour les analyses de laboratoire. L'étude au laboratoire a conduit 
Tableau 1. Caractéristiques des palmeraies étudiées - Characteristics of the studied palm plantations.

\begin{tabular}{|c|c|c|c|c|c|c|}
\hline $\begin{array}{l}\mathrm{N}^{\circ} \\
\text { palmeraie }\end{array}$ & $\begin{array}{l}\text { Coordonnées } \\
\text { du centre de }\end{array}$ & $\begin{array}{l}\text { ographiques } \\
\text { parcelle }\end{array}$ & $\begin{array}{l}\text { Superficie } \\
\text { (ha) }\end{array}$ & $\begin{array}{l}\text { Âge de la plantation } \\
\text { (an) }\end{array}$ & Classe d'âge & $\begin{array}{l}\text { Durée de gestion } \\
\text { des feuilles (an) }\end{array}$ \\
\hline 1 & $6^{\circ} 49^{\prime} 26,1 ” \mathrm{~N}$ & $2^{\circ} 38^{\prime} 25,2^{\prime \prime} \mathrm{E}$ & 2 & 11 & Pré-adulte & 4 \\
\hline 2 & $6^{\circ} 47^{\prime} 59,4{ }^{\prime \prime} \mathrm{N}$ & $2^{\circ} 37^{\prime} 01,6{ }^{\prime \prime} \mathrm{E}$ & 2 & 9 & Pré-adulte & 2 \\
\hline 3 & $6^{\circ} 49^{\prime} 39,1$ " N & $2^{\circ} 38^{\prime} 51,99^{\prime \prime} \mathrm{E}$ & 3 & 10 & Pré-adulte & 3 \\
\hline 4 & $6^{\circ} 49^{\prime} 45,1 ” \mathrm{~N}$ & $2^{\circ} 38^{\prime} 29,3{ }^{\prime \prime} \mathrm{E}$ & 4 & 17 & Adulte & 10 \\
\hline 5 & $6^{\circ} 45^{\prime} 35,0^{\prime \prime} \mathrm{N}$ & $2^{\circ} 37^{\prime} 38,2$ '” & 7 & 17 & Adulte & 10 \\
\hline 6 & $6^{\circ} 59^{\prime} 06,3^{\prime \prime} \mathrm{N}$ & $2^{\circ} 40^{\prime} 34,7^{\prime \prime} \mathrm{E}$ & 2,5 & 18 & Adulte & 11 \\
\hline
\end{tabular}

à l'obtention des teneurs des propriétés du sol sur les 360 échantillons. La méthode analytique adoptée a consisté en l'utilisation de la spectrométrie proche infrarouge pour la prédiction des teneurs avec des modèles de régression établis sur la base de résultats d'analyses physico-chimiques classiques faites sur un nombre plus réduit d'échantillons. Le principe a été basé sur les étapes suivantes :

- choix des échantillons de calibration et de validation des modèles de prediction. Le passage de tous les échantillons au spectromètre a permis d'acquérir leurs données spectrales qui ont été analysées séparément avec le logiciel WinISI III-v1.61e (Foss NIRSystems/ Tecator International, LLC, Silver Spring, MD, USA). Une analyse en composantes principales (ACP) a été réalisée sur les absorbances. Une centaine d'échantillons (exactement 101 échantillons dans cette étude) les plus représentatifs ont été sélectionnés par le logiciel pour constituer le lot d'échantillons de calibration. La sélection se base sur le concept du voisinage (distance entre deux échantillons voisins) dans l'espace des composantes principales. Par la suite, 30 échantillons ont été sélectionnés sur les 259 échantillons restants pour constituer le lot d'échantillons de validation ;

- analyses physico-chimiques des échantillons de sol. Les échantillons sélectionnés pour la calibration et la validation des modèles de prédiction (131 échantillons) ont été analysés au laboratoire selon les méthodes conventionnelles décrites par Pansu et al. (2006). Les teneurs en carbone et azote organiques ont été déterminées par la méthode du CHN par combustion sèche totale du sol sous oxygène avec comme gaz vecteur l'hélium pur à $870^{\circ} \mathrm{C}$. Le $\mathrm{pH}$ du sol a été déterminé au moyen d'un pHmètre après une mise en solution aqueuse du sol pendant $2 \mathrm{~h}$, dans un rapport sol/eau de $1 / 2,5$. Une extraction à l'acétate d'ammonium $(1 \mathrm{~N}, \mathrm{pH}=7)$ dans un rapport sol/solution de $1 / 20$, puis un lavage du sol par le chlorure de potassium (1N) pour déplacer en solution les ions $\mathrm{NH}_{4}^{+}$, ont permis de déterminer les cations échangeables, calcium $(\mathrm{Ca})$, magnésium $(\mathrm{Mg})$ et potassium $(\mathrm{K})$ et la capacité d'échange cationique (CEC). Les cations échangeables sont dosés dans l'extrait d'acétate d'ammonium au moyen d'un spectromètre d'émission plasma (ICP). Les ions $\mathrm{NH}_{4}^{+}$déplacés en solution représentent la CEC et sont dosés par colorimétrie ;

- prédiction des propriétés du sol. Les résultats des 131 analyses conventionnelles ont servi à établir des modèles de régression pls (partial least square) pour prédire les propriétés du sol sur les autres échantillons (Barthès et al., 2006 ; Brunet et al., 2007). La précision des modèles de prédiction par la SPIR a été appréciée en calibrant le modèle à partir des analyses des 101 échantillons de calibration et en le validant sur les données d'analyses des 30 échantillons restants. Les paramètres suivants ont été examinés : $\mathrm{R}_{\text {Cal }}^{2}$ (coefficient de détermination de la calibration des modèles), $\mathrm{R}_{\text {val }}^{2}$ (coefficient de validation des modèles) et $\mathrm{RPD}_{\mathrm{cv}}^{\text {val (ratio de }}$ déviation de la performance de validation croisée). La prédiction est d'autant meilleure que $\mathrm{R}_{\text {Cal }}^{2}$ et $\mathrm{R}^{2}$ val sont proches de 1 et selon Chang et al. (2001), la validation croisée des modèles est bonne si $\mathrm{RPD}_{\mathrm{cv}}>$ 2 , elle est moyenne si $1,4<\mathrm{RPD}_{\mathrm{cv}}<2$ et mauvaise si $\mathrm{RPD}_{\mathrm{cv}}<1,4$. Le tableau 2 présente les valeurs obtenues pour les modèles retenus pour la prédiction des propriétés de nos échantillons.

\subsection{Traitement des données}

Calcul de la densité apparente, de la porosité et des stocks de carbone et d'azote du sol. La densité apparente du sol désigne la masse volumique du sol. Elle a été déterminée par le rapport de la masse sèche du sol par le volume du cylindre de prélèvement. Les valeurs de densité apparente du sol ont servi à calculer la porosité du sol selon la formule :

$$
\text { Porosité }(\%)=(1-\mathrm{Da} / \mathrm{Dr}) * 100
$$

où $\mathrm{Da}$ est la densité apparente du sol et Dr la densité réelle du sol. Pour Dr, nous avons retenu la valeur de 2,65 dont Aho et al. (1997) ont montré qu'elle représentait bien celle des sols ferrallitiques. 
Tableau 2. Paramètres de calibration, de validation croisée et de validation externe des modèles de prédiction des propriétés du sol par la méthode de la spectroscopie proche infrarouge basée sur des régressions pls (partial least square) - Parameters of calibration, cross validation and external validation of the models of soil properties prediction established by partial least square regressions ( $\mathrm{pls}$ ) on near infrared spectroscopy data.

\begin{tabular}{|c|c|c|c|c|c|c|c|c|c|c|}
\hline \multirow[t]{2}{*}{ Propriété du sol } & \multirow{2}{*}{$\begin{array}{l}\text { Meilleur pré- } \\
\text { traitement } \\
\text { spectral }\end{array}$} & \multicolumn{2}{|c|}{$\begin{array}{l}\text { Calibration } \\
\text { pls }\end{array}$} & \multicolumn{2}{|c|}{$\begin{array}{l}\text { Validation croisée } \\
\text { pls }\end{array}$} & \multicolumn{4}{|c|}{ Validation externe pls } & \multirow{2}{*}{$\begin{array}{l}\text { Précision de } \\
\text { la prédiction } \\
\text { selon Chang } \\
\text { et al. (2001) }\end{array}$} \\
\hline & & N1 & $\mathrm{SE}_{\mathrm{CV}}$ & $\mathrm{R}_{\mathrm{CV}}^{2}$ & $\mathrm{RPD}_{\mathrm{CV}}$ & $\mathrm{N} 2$ & SEP & $\mathrm{R}_{\mathrm{val}}^{2}$ & $\mathrm{RPD}_{\mathrm{val}}$ & \\
\hline $\mathrm{C}_{\text {org }}\left(\mathrm{g} \cdot \mathrm{kg}^{-1}\right)$ & Std MSC 0011 & 95 & 1,50 & $0,97 * * *$ & 4,9 & 30 & 2,74 & $0,91 * * *$ & 3,0 & Bonne \\
\hline $\mathrm{N}_{\text {tot }}\left(\mathrm{g} \cdot \mathrm{kg}^{-1}\right)$ & Std MSC 0011 & 94 & 0,11 & $0,95 * * *$ & 3,8 & 30 & 0,20 & $0,89^{* * *}$ & 2,6 & Bonne \\
\hline $\mathrm{pH}_{\text {eau }}$ & Detrend 1441 & 99 & 0,28 & $0,81 * * *$ & 1,9 & 30 & 0,24 & $0,72 * * *$ & 1,9 & Moyenne \\
\hline $\mathrm{Ca}\left(\mathrm{meq} \cdot 100 \mathrm{~g}^{-1}\right)$ & Std MSC 1441 & 94 & 0,44 & $0,94 * * *$ & 3,0 & 30 & 0,68 & $0,87 * * *$ & 2,5 & Bonne \\
\hline $\operatorname{Mg}\left(\right.$ meq $\left.\cdot 100 \mathrm{~g}^{-1}\right)$ & Std MSC 1441 & 95 & 0,29 & $0,89 * * *$ & 2,5 & 30 & 0,39 & $0,78^{* * *}$ & 2,1 & Bonne \\
\hline $\mathrm{K}\left(\mathrm{meq} \cdot 100 \mathrm{~g}^{-1}\right)$ & SNV 1441 & 93 & 0,02 & $0,88 * * *$ & 2,1 & 30 & 0,01 & $0,80 * * *$ & 2,1 & Bonne \\
\hline $\mathrm{S}\left(\mathrm{meq} \cdot 100 \mathrm{~g}^{-1}\right)$ & Std MSC 0011 & 95 & 0,76 & $0,93 * * *$ & 3,0 & 30 & 0,88 & $0,90 * * *$ & 2,9 & Bonne \\
\hline $\left.\operatorname{CEC~(meq.100~g~}{ }^{-1}\right)$ & SNV 0011 & 94 & 0,87 & $0,91 * * *$ & 2,8 & 30 & 1,19 & $0,77 * * *$ & 2,1 & Bonne \\
\hline
\end{tabular}

Pré-traitement spectral - spectra pre-treatments : Std MSC $0011=$ standard multiplicative scatter correction; $1^{\mathrm{er}}$ chiffre : ordre de la dérivée, $2^{\mathrm{e}}$ chiffre : nombre de longueurs d'ondes sur lesquelles la dérivation a été faite, $3^{\mathrm{e}}$ et $4^{\mathrm{e}}$ chiffres : niveau de lissage du modèle $-1^{\text {st }}$ digit: derivative order, $2^{\text {nd }}$ digit: number of wavelengths over which the derivative is applied, $3^{\text {rd }}$ and $4^{\text {th }}$ digits: model smoothing levels ; SNV : standard normal variate; N1 : nombre d'échantillons utilisés pour la calibration des modèles après élimination des échantillons déviants. Le nombre d'échantillons retenus au départ pour la calibration est de 101 - number of samples after the elimination of calibration outliers, 101 samples were choosen for calibration; N2 : nombre d'échantillons utilisés pour la validation externe - number of samples used for the external validation; $\mathrm{SE}_{\mathrm{CV}}, \mathrm{SEP}$ : respectivement erreurs standard de la validation croisée et de la prédiction - standard errors of cross validation and prediction, respectively; $\mathrm{R}_{\mathrm{CV}}^{2}, \mathrm{R}_{\mathrm{val}}^{2}$ : coefficients de détermination de la validation croisée et de la validation externe - coefficients of determination of cross validation and external validation; $\mathrm{RPD}_{\mathrm{CV}}$, $\mathrm{RPD}_{\text {val }}$ : ratios de déviation de la performance de la validation croisée et de la validation externe - ratios of performance to deviation for cross validation and external validation; $* * *$ : significatif à $p<0,01-$ significant at $\mathrm{p}<0.01$.

Les valeurs de Da et les teneurs en carbone et en azote ont été utilisées pour calculer les stocks de carbone et d'azote par masse de terre équivalente (MTE) pour les premiers $650 \mathrm{~kg} \cdot \mathrm{m}^{-2}$ de sol. Cette valeur est proche de la masse totale de terre fine calculée sur la profondeur de $50 \mathrm{~cm}$ sous andain ou dans l'interligne. Le calcul de la masse totale de terre fine et des stocks de carbone et d'azote a été opéré selon la procédure suivante tirée des travaux de Ellert et al. (1995) et Ellert et al. (2002) :

- on a déterminé que l'on pouvait atteindre une MTE de $650 \mathrm{~kg} \cdot \mathrm{m}^{-2} \mathrm{de}$ sol pour toutes les fosses en utilisant les données des cinq couches de sol ;

- pour chaque fosse, on a calculé l'épaisseur exacte de la couche la plus profonde pour atteindre exactement la MTE, puis on a calculé l'épaisseur totale exacte (Ec) correspondante ;

- enfin, on a calculé le stock de C pour l'épaisseur Ec de sol en cumulant les stocks $\mathrm{C}_{\mathrm{i}}$ de chaque couche.

Le stock de C (QC) par MTE a été obtenu par la formule suivante :

$$
\mathrm{QC}=\sum_{i=1}^{i=5} C_{i} \times D a_{i} \times E_{i} \times T F_{i}
$$

avec QC en $\mathrm{Mg} \cdot \mathrm{ha}^{-1}$; teneurs pondérales en carbone $C_{i}$ en $\mathrm{g} \cdot 100 \mathrm{~g}^{-1}$; densité apparente $D a_{i}$ en $\mathrm{g} \cdot \mathrm{cm}^{-3}$ (ou kg. $\left.\mathrm{dm}^{-3}\right)$; épaisseurs $E_{i}$ en $\mathrm{cm}$ pour la couche $i$; taux de terre fine $T F_{i}$ en $\mathrm{g} \cdot \mathrm{g}^{-1}$.

Analyse statistique. Les données de la première phase de l'étude (stocks de carbone des six parcelles de deux âges) ont permis d'étudier les variations de stock de carbone et d'azote par MTE pour RT et pour RN des palmeraies des deux classes d'âges. Les données de la deuxième phase ont permis de comparer les propriétés physico-chimiques des sols pour RT et RN dans les parcelles adultes.

Dans les deux phases de l'étude, l'effet sur les propriétés du sol des facteurs étudiés (âge, mode de gestion des feuilles et profondeur), les différences entre plantations d'une même classe d'âge et la variabilité aléatoire liée à chaque niveau d'observation (arbre, fosse et prélèvement), ont été évalués et testés en appliquant un Modèle Linéaire Mixte (PROC MIXED, SAS Institute 2011).

Pour la première phase (étude du stock de $\mathrm{C}$ et $\mathrm{N}$ en fonction de l'âge et du mode de gestion), le modèle a intégré les variabilités aléatoires liées à l'effet de chaque arbre dans la parcelle de plantation et à l'interaction «parcelle $\mathbf{x}$ mode de restitution». Les résidus calculés pour chacun des deux stocks mesurés autour d'un même arbre (l'un dans l'andain et l'autre 
dans l'interligne) ont été considérés comme des erreurs aléatoires indépendantes, conditionnellement à l'effet de l'arbre.

Pour la deuxième phase, l'effet aléatoire de chaque arbre a été inclus dans le modèle et les résidus calculés pour chacun des prélèvements dans une fosse ont été modélisés par un processus autorégressif d'ordre 1 qui introduit une corrélation entre les mesures faites à des niveaux contigus (Littell et al., 2006).

Les interactions entre facteurs étudiés ont été détaillées en testant l'effet de chaque facteur par tranches de combinaison des niveaux des autres facteurs (tests connus sous le nom de «tests of simple effects » [SAS Institute, 2011]). Des comparaisons multiples de moyennes par le test de Tukey ont permis d'identifier les différences significatives entre paires. Les comparaisons ont été faites entre andain et interligne et par profondeur.

\section{RÉSULTATS}

\subsection{Stocks de carbone et d'azote dans le sol}

Les stocks de carbone et d'azote pour $650 \mathrm{~kg} \cdot \mathrm{m}^{-2}$ de terre (environ $50 \mathrm{~cm}$ de profondeur de sol) sont présentés pour chaque modalité de restitution et classe d'âge (Figure 2). Les stocks de carbone (58,0 Mg.ha $\left.{ }^{-1}\right)$ et d'azote $\left(4,3 \mathrm{Mg} \cdot \mathrm{ha}^{-1}\right)$ obtenus avec RT en palmeraies adultes sont significativement $(p<0,001)$ supérieurs aux stocks obtenus pour toutes les autres situations dont les valeurs varient autour d'une moyenne de $34,6 \mathrm{Mg}$. $\mathrm{ha}^{-1}$ et $2,9 \mathrm{Mg} \cdot \mathrm{ha}^{-1}$ respectivement pour le carbone et l'azote. Il n'y a pas de différence significative au seuil de $5 \%$ entre les stocks sous RT et RN à l'âge pré-adulte, ni entre les stocks sous RN des deux classes d'âges.

\subsection{Propriétés chimiques du sol des palmeraies adultes}

Pour l'ensemble des variables examinées, les teneurs et propriétés chimiques des sols décroissent avec la profondeur (Annexe 1). Au sein d'une même zone, les teneurs des horizons $0-5 \mathrm{~cm}, 5-10 \mathrm{~cm}$ et $10-20 \mathrm{~cm}$ sont fréquemment significativement différentes entre elles et presque toujours significativement supérieures à celles des horizons plus profonds. Le $\mathrm{pH}$ de l'horizon $0-5 \mathrm{~cm}$ n'est supérieur à celui des horizons plus profonds que sous RT et il ne présente aucune variation significative avec la profondeur sous RN.

La comparaison entre zones pour un même horizon montre aussi que les teneurs en $\mathrm{C}, \mathrm{N}$ et les cations sont significativement plus élevées sous RT jusqu'à une profondeur de $20 \mathrm{~cm}$. On observe les mêmes effets statistiques jusqu'à $20 \mathrm{~cm}$ pour la somme des cations échangeables (S) et la capacité d'échange cationique (CEC).

La figure 3 illustre les variations observées avec la profondeur et par zone, pour quatre variables importantes de la fertilité des sols sous palmeraie : $\mathrm{C}$ total, $\mathrm{N}$ total, $\mathrm{pH}$ et $\mathrm{K}$. Elle montre une décroissance des propriétés du sol avec la profondeur et confirme qu'au-delà de $20 \mathrm{~cm}$, les écarts entre RT et RN sont minimes.

\subsection{Densité apparente et porosité du sol dans les palmeraies adultes}

La densité apparente augmente avec la profondeur du sol, que ce soit avec RT ou avec RN. Pour les deux
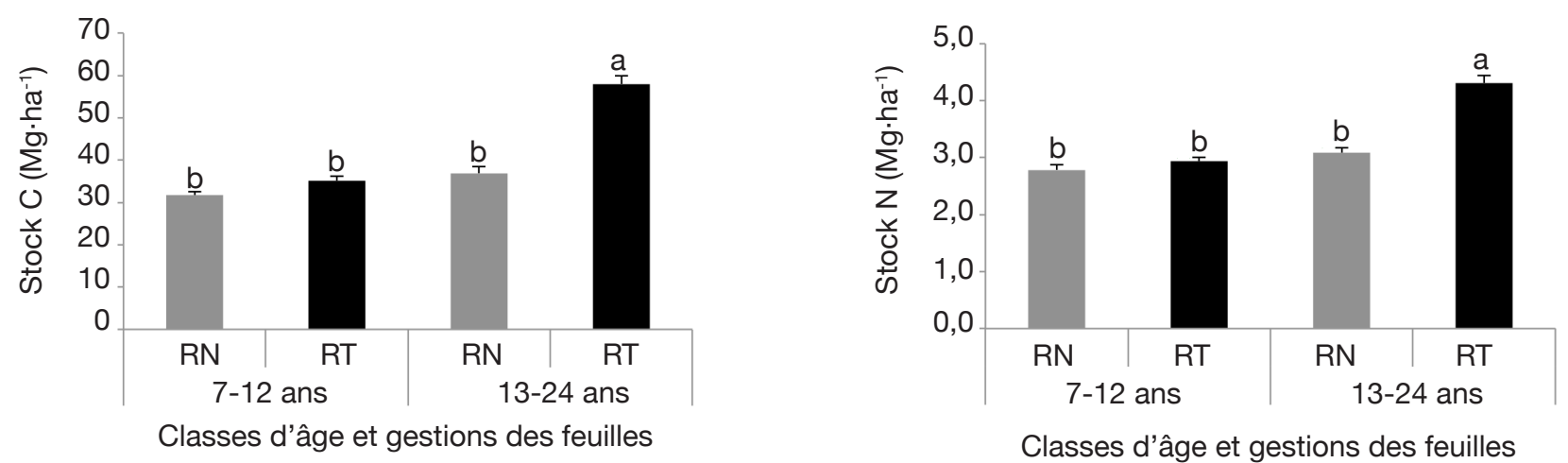

Figure 2. Stocks de carbone et d'azote pour $650 \mathrm{~kg} \cdot \mathrm{m}^{-2}$ de masse de terre équivalente $(\sim 0-50 \mathrm{~cm})$ pour les traitements restitution totale (RT) et restitution nulle $(\mathrm{RN})$ des palmeraies de 7-12 ans et 13-24 ans - Carbon and nitrogen stocks for $650 \mathrm{~kg} \cdot \mathrm{m}^{-2}$ of equivalent soil mass $(\sim 0-50 \mathrm{~cm})$ for the total recycling $(T R)$ and no recycling $(N R)$ treatments of oil palm plantation of 7-12 and 13-24 year-old.

Les moyennes sont présentées avec les barres d'erreur standard - the mean values are presented with standard error bars; les lettres a et $\mathrm{b}$ indiquent le classement des moyennes selon le test de Tukey $(p<5 \%)$ - the letters $a$ and $b$ indicate the classification of the mean values according to Tukey test $(\mathrm{p}<5 \%)$. 

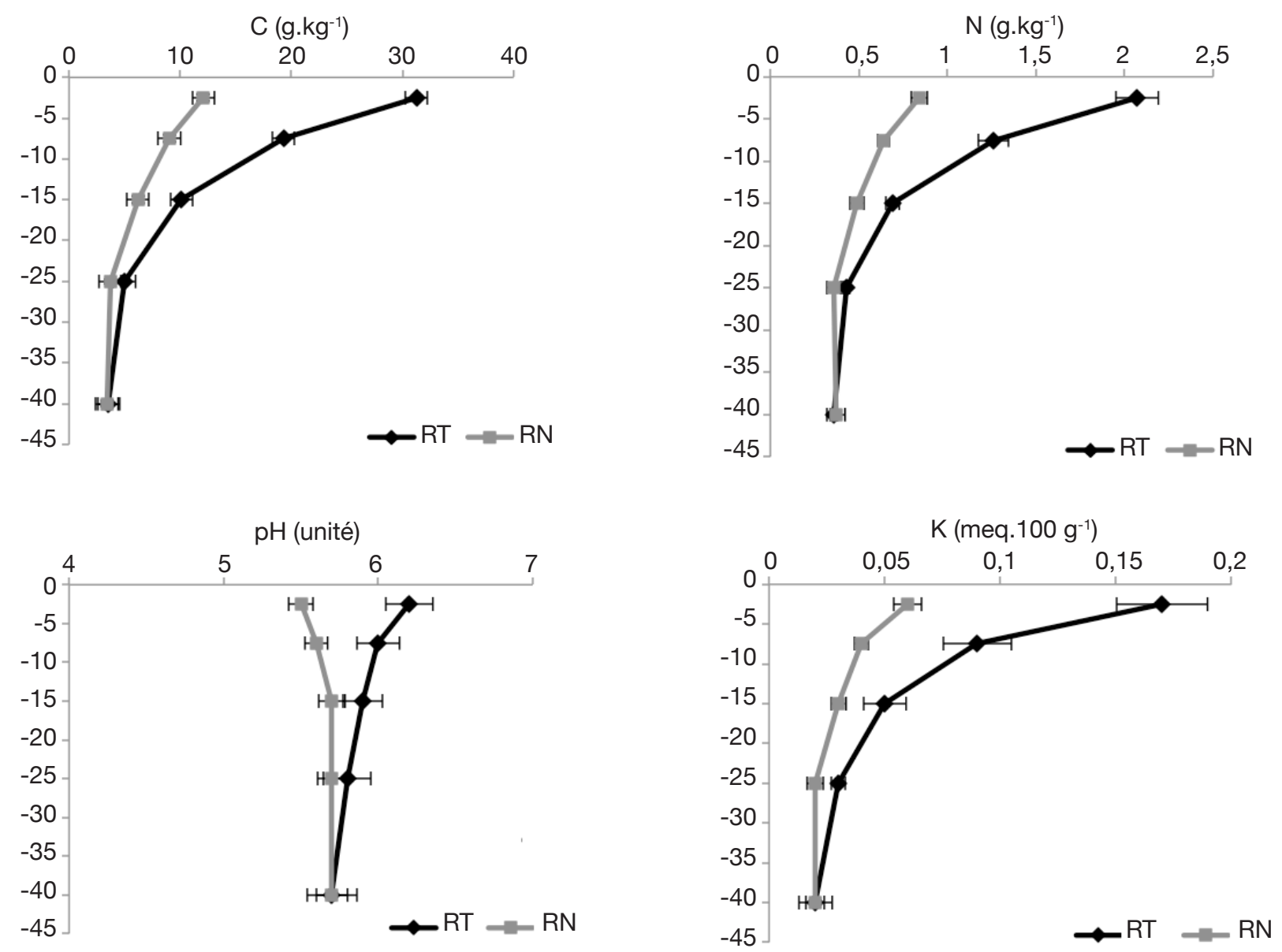

Figure 3. Illustration de l'effet des modalités de recyclage des feuilles d'élagage (RT, restitution totale ; RN, restitution nulle) sur les paramètres physico-chimiques du sol - Illustration of the effect of the recycling modes of pruned oil palm fronds (RT, total recycling; $R N$, no recycling) on the soil chemical parameters.

Chaque moyenne est présentée avec l'intervalle de confiance à $95 \%$ - each mean is presented with the confidence interval to $95 \%$.

modalités, les écarts entre deux horizons de surface sont significatifs entre eux et par rapport aux trois horizons profonds qui restent confondus (Figure 4). Les valeurs de densité apparente obtenues pour RT sont systématiquement inférieures à celles de $\mathrm{RN}$ et les écarts sont significatifs sur toute la profondeur étudiée.

La porosité totale du sol diminue avec la profondeur du sol. On obtient évidemment les mêmes différences entre horizons que pour la densité du sol (Tableau 3). Tout au long du profil, la porosité du sol sous RT est significativement supérieure à celle de RN. Les différences sont importantes sur les deux premiers horizons (respectivement 17 et 9\%), puis elles diminuent en profondeur où elles varient peu, autour de $4 \%$ en moyenne.

\section{DISCUSSION}

Dans cette étude qui vise à évaluer l'effet du recyclage des feuilles élaguées sur la matière organique et sur la fertilité du sol dans les palmeraies villageoises, on constate qu'après dix années de recyclage, les stocks de $\mathrm{C}$ et $\mathrm{N}$ sont significativement plus élevés sous RT par rapport à RN. Ils ne le sont pas pour les palmeraies pré-adultes où les feuilles sont recyclées pendant seulement une moyenne de trois années. On constate aussi que les stocks de $\mathrm{C}$ et $\mathrm{N}$ ont augmenté respectivement de $65 \%$ et $47 \%$ entre les deux classes d'âge pour RT, mais seulement de $16 \%$ et $11 \%$ entre les deux classes d'âge pour RN. On peut donc penser que c'est bien la durée d'accumulation des feuilles au sol qui conduit à augmenter progressivement le stock de matière organique du sol (MOS) jusqu'à ce que la différence entre RT et RN devienne significative. Ceci est cohérent avec les observations de Frazão et al. (2013) qui n'avaient pas trouvé de différence significative entre les stocks de carbone sous RT $\left(27,4 \mathrm{Mg} \mathrm{C} \cdot \mathrm{ha}^{-1}\right)$ et sous RN (27,0 Mg C.ha- $\left.{ }^{-1}\right)$ sur une profondeur de $30 \mathrm{~cm}$ de sol dans des palmeraies brésiliennes de 8 ans, encore trop jeunes. Nos résultats confirment l'existence d'une variabilité à la fois spatiale et temporelle des stocks de 


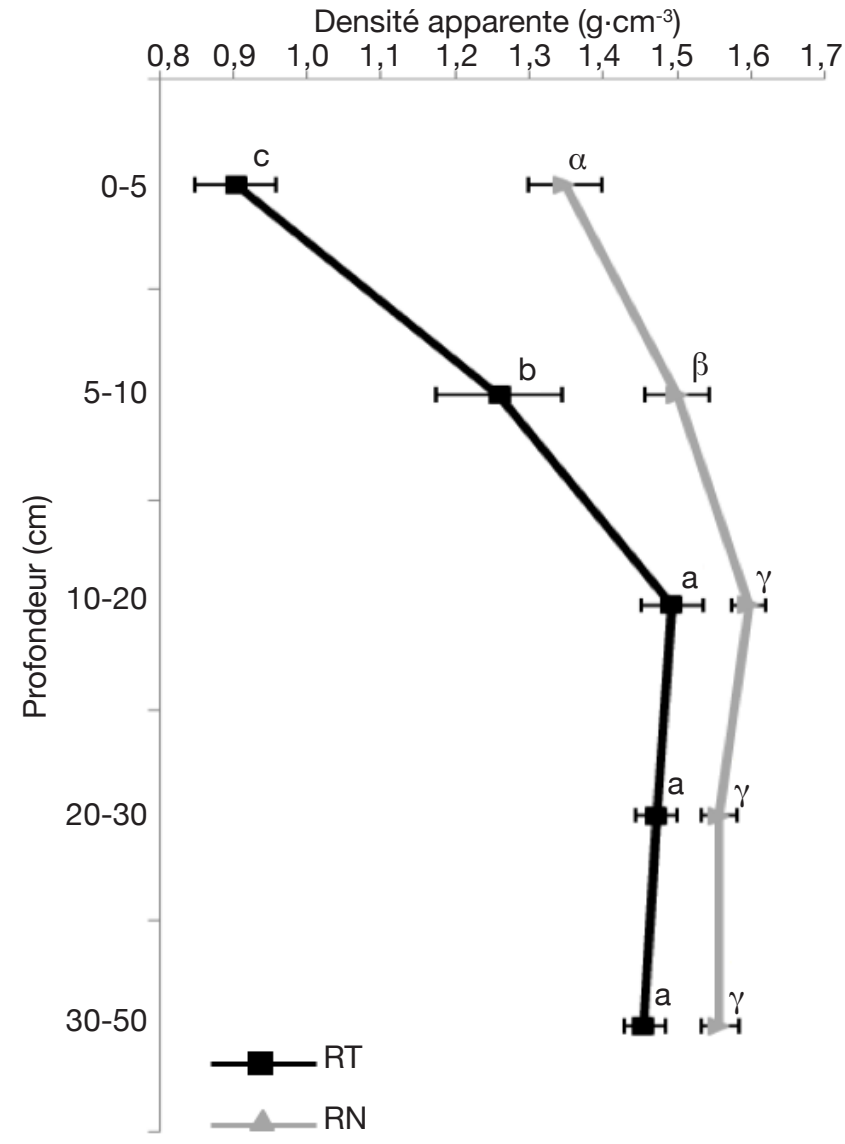

Figure 4. Évolution de la densité apparente du sol sous restitution totale (RT) et restitution nulle $(\mathrm{RN})$ jusqu'à $50 \mathrm{~cm}$ de profondeur du sol dans les palmeraies adultes (13-24 ans) - Evolution of the soil bulk density under total recycling $(R T)$ and no recycling $(R N)$ for $0-50 \mathrm{~cm}$ soil layer in adult palm (13-24 years).

Les barres d'erreurs sont placées au niveau de chaque point pour indiquer l'intervalle de confiance à $95 \%$ - Each mean is presented with the confidence interval to $95 \%$; les lettres indiquent la comparaison des moyennes par le test de Tukey pour les modalités RT (lettres romaines) et RN (lettres grecques) - the letters indicate the comparison of means by Tukey test for $T R$ (Roman letters) and NR (Greek letters) modes.

MOS, qui avait été signalée par d'autres auteurs (Djègui, 1992 ; Law et al., 2009). Frazão et al. (2013) ont aussi montré des différences de stocks de carbone entre RT $\left(29,6 \mathrm{Mg} \cdot \mathrm{ha}^{-1}\right)$ et $\mathrm{RN}\left(25,4 \mathrm{Mg} \cdot \mathrm{ha}^{-1}\right)$ dans des palmeraies de 25 ans au Brésil. Ils ont aussi constaté en 2014 que le stock de carbone sous andains de feuilles d'élagage d'une palmeraie de 34 ans était supérieur de $78 \%$ à celui d'une plantation de 23 ans (Frazão et al., 2014).

En considérant que RT ne représente que $10 \%$ de la surface agricole, on peut calculer à partir de la masse de terre équivalente (MTE) de $650 \mathrm{~kg} \cdot \mathrm{m}^{-2}$, le stock moyen de $\mathrm{C}$ d'une palmeraie adulte où les feuilles d'élagage sont recyclées. On obtient une valeur de $39 \mathrm{Mg} \cdot \mathrm{ha}^{-1}$ pour une profondeur de sol d'environ $50 \mathrm{~cm}$. Le gain est de
Tableau 3. Porosité moyenne du sol (\%) par profondeur sous restitution totale (RT) et restitution nulle (RN) dans les palmeraies adultes - Soil porosity (\%) for the soil layers under total recycling $(R T)$ and no recycling $(R N)$ in adult oil palm plantations.

\begin{tabular}{cll}
\hline Profondeur $(\mathrm{cm})$ & Porosité sol RT & Porosité sol RN \\
\hline $0-5$ & $66 \pm 4^{\mathrm{a}, \alpha}$ & $49 \pm 4^{\mathrm{a}, \beta}$ \\
\hline $5-10$ & $52 \pm 7^{\mathrm{b}, \alpha}$ & $43 \pm 4^{\mathrm{b}, \beta}$ \\
$10-20$ & $44 \pm 3^{\mathrm{c}, \alpha}$ & $40 \pm 2^{\mathrm{c}, \beta}$ \\
\hline $20-30$ & $44 \pm 2^{\mathrm{c}, \alpha}$ & $41 \pm 2^{\mathrm{c}, \beta}$ \\
$30-50$ & $45 \pm 2^{\mathrm{c}, \alpha}$ & $41 \pm 2^{\mathrm{c}, \beta}$ \\
\hline
\end{tabular}

Les valeurs moyennes de porosité sont présentées \pm leur écarttype - mean values of soil porosity averages are presented \pm the standard deviation; les lettres en exposant de chaque valeur indiquent la comparaison des moyennes selon le test de Tukey - superscript letters indicate the comparison of the means by the Tukey test; les lettres romaines comparent les moyennes des profondeurs dans un même mode de gestion des feuilles d'élagage, alors que les lettres grecques comparent les moyennes d'une même profondeur entre les deux modes de gestion - Roman letters compare the mean values of the same mode of pruned fronds management, while the Greek letters compare the same soil layer between the two modes.

2,1 Mg.ha-1 ${ }^{-1}$, soit 5,7\% par rapport à une palmeraie du même âge où l'on ne recyclerait pas les feuilles. Un calcul analogue pour l'azote conduit à un stock total de 3,21 Mg.ha- ${ }^{-1}$ dans une palmeraie RT, soit un gain de $0,12 \mathrm{Mg} \cdot \mathrm{ha}^{-1}$, qui représente $4 \%$ par rapport à une palmeraie sans recyclage des feuilles. Notre stock de carbone est cohérent avec les $34,3 \mathrm{Mg} \cdot \mathrm{ha}^{-1}$ indiqués par Frazão et al. (2014) dans les palmeraies industrielles de 23 ans au Brésil, pour une profondeur de $30 \mathrm{~cm}$ du sol.

Nos résultats confirment que les palmeraies villageoises du Bénin seraient, à l'instar d'autres cultures, un «puits de carbone » que le recyclage de la biomasse peut alimenter (Arrouays et al., 2002 ; Bernoux et al., 2011). L'augmentation des stocks de carbone dans les palmeraies a été identifiée par Carlson et al. (2012) comme un moyen de mitigation des émissions de gaz à effet de serre dans certaines conditions de précédents et de pratiques culturaux.

Notre estimation pour une palmeraie adulte est proche du stock de carbone $\left(42 \mathrm{Mg} \cdot \mathrm{ha}^{-1}\right)$ trouvé par Barthès et al. (2004) sur 0-40 cm de profondeur du sol dans le système agricole maïs-mucuna installé sur une terre de barre du sud-Bénin, avec restitution des résidus du mucuna pendant 10 ans. Ce qui confirme que le potentiel de stockage des palmeraies est aussi important que d'autres cultures. Notre estimation est toutefois inférieure à celles mesurées dans des forêts secondaires par Djegui et al. (1992) au Bénin (48 Mg C.ha ${ }^{-1}$ sur 0-35 cm) et par Lardy Chapuis et al. (2002) au Brésil (53 Mg C.ha ${ }^{-1}$ sur 0-20 cm). 
Cette réflexion indique que l'intérêt des palmeraies pour la séquestration du carbone doit donc être modulé en fonction du précédent cultural. Reinhardt et al. (2007) ont montré que lorsque les palmeraies ne sont pas directement plantées après une forêt primaire, le stock de carbone du sol augmente avec le temps en raison du recyclage de la biomasse aérienne. Les palmeraies béninoises dans leur grande majorité sont implantées sur d'anciennes cultures annuelles (Aholoukpè et al., 2013) qui ont contribué à l'appauvrissement en carbone du sol (Amadji et al., 2008). Elles peuvent donc être très efficaces pour restaurer les stocks de $\mathrm{C}$ lorsque les feuilles ne sont pas exportées.

L'augmentation des stocks de matière organique du sol avec RT en palmeraies adultes a eu des effets positifs sur les propriétés physico-chimiques du sol. Sur les 20 premiers $\mathrm{cm}$, les écarts entre RT et RN pour la porosité du sol, la CEC, les cations et les teneurs en $\mathrm{N}$, sont significatifs. On a noté aussi une amélioration de 0,7 unité de $\mathrm{pH}$ sur l'horizon $0-5 \mathrm{~cm}$. Ces résultats confirment les effets positifs signalés par Goh et al. (2003) en Asie du Sud-Est et par Nelson et al. (2011) en Papouasie-Nouvelle-Guinée avec le recyclage des rafles en replantation: amélioration de la CEC, des cations échangeables et du $\mathrm{pH}$ du sol.

Ces changements dus au recyclage des feuilles sont d'importance puisqu'ils touchent des propriétés qui conditionnent directement les facteurs de productivité du palmier à huile :

$-\mathrm{N}$ et $\mathrm{K}$ sont deux éléments essentiels dans les processus métaboliques qui contrôlent la production de matière sèche $(\mathrm{N}$ : assimilation chlorophyllienne, $\mathrm{K}$ : effet sur la photosynthèse via le contrôle de l'ouverture stomatique, transport des carbohydrates vers les régimes) (Nodichao, 2008 ; Dubos et al., 2011) ;

- les réserves hydriques du sol sont améliorées par l'augmentation de la porosité concomitante à l'augmentation des teneurs en MOS, celles de $\mathrm{Ca}$ et $\mathrm{Mg}$ ainsi que la CEC qui favorisent l'obtention d'un complexe argilo-humique stable conférant au sol une meilleure structure.

Ces résultats montrent que le choix que nous avons fait pour une MTE de $650 \mathrm{~kg} \cdot \mathrm{m}^{-2}$ est donc justifié puisqu'en travaillant sur une profondeur qui n'excédait pas $50 \mathrm{~cm}$, nous avons largement couvert la couche de sol où se produisent les changements majeurs induits par le recyclage des feuilles.

Ces effets intéressent certes un volume de sol limité. Cependant, sous l'andain, à mi-distance entre deux palmiers, l'essentiel de la biomasse des racines est située dans les 30 premiers $\mathrm{cm}$ du sol et en particulier les racines fines absorbantes sont situées dans les 10 premiers $\mathrm{cm}$ (Nodichao et al., 2008). La surface traitée ne représente que $10 \%$ de la surface colonisée par les racines d'une palmeraie adulte, mais le progrès mérite d'être souligné si on compare les propriétés du sol RN aux normes qui prévalent dans la profession pour l'horizon de surface: Goh et al. ${ }^{2}$ (1997), cités par Paramananthan (2003), considèrent en effet que des limitations du rendement sont attendues lorsque les seuils suivants ne sont pas atteints : $\mathrm{C}: 15 \mathrm{~g} \cdot \mathrm{kg}^{-1}$, $\mathrm{N}: 1,5 \mathrm{~g} \cdot \mathrm{kg}^{-1}$, $\mathrm{K}$ et $\mathrm{Mg}: 0,25 \mathrm{meq} \cdot 100 \mathrm{~g}^{-1}$. Les teneurs en $\mathrm{C}, \mathrm{N}$ et $\mathrm{K}$ des 20 premiers centimètres du sol de la modalité $\mathrm{RN}$ sont donc très faibles en comparaison avec ces normes. La CEC est aussi très faible si on compare avec les normes optimales données par Paramananthan (2003) (18 meq.100 g $\mathrm{g}^{-1}$ ) ou Nelson et al. (2010).

Compte tenu de ces considérations, dans le contexte de plantations dans des conditions de faible fertilité, il est donc prudent de ne pas se priver d'une surface, même réduite comme celle de recyclage des feuilles, où la fertilité du sol est beaucoup plus proche des conditions minimales recommandées pour la culture. Nous n'avons toutefois pas pu corroborer par des observations de production, réalisées en nombre insuffisant (données non présentées), que le recyclage des feuilles aurait un impact direct sur les rendements des plantations de notre étude.

\section{CONCLUSIONS}

Le recyclage des feuilles d'élagage induit au fil des années des modifications des propriétés des sols sous les zones de restitution. Nous avons pu en mesurer les effets significatifs sur les stocks de $\mathrm{C}$ et $\mathrm{N}$ après 10 ans de recyclage dans les palmeraies adultes. L'augmentation de la MOS améliore aussi les propriétés physicochimiques du sol par rapport aux zones non traitées. Les effets positifs sur les teneurs en éléments nutritifs ( $\mathrm{N}, \mathrm{K}, \mathrm{Ca}, \mathrm{Mg}$ ), la porosité du sol et sa CEC sont significatifs jusqu'à une profondeur de $20 \mathrm{~cm}$, or cette couche de sol est très colonisée par les racines. Même si la surface couverte par les andains ne représente qu'environ $10 \%$ de la surface cultivée, nous pensons qu'elle peut jouer un rôle favorable pour la culture, ne serait-ce qu'en raison des conditions de fertilité très médiocres que nous avons trouvées pour le sol hors recyclage. On peut conclure qu'il est recommandé de ne pas exporter les feuilles d'élagage, d'autant plus que l'espace amendé sera utilisé pour planter la prochaine génération de palmiers après 25 ans environ.

\section{Bibliographie}

Adjadi E., 2012. Le développement du palmier à huile sélectionné au Bénin. Rapport d'étude. Actualisation des

\footnotetext{
${ }^{2}$ Goh K.J. et al., 1997. Developing decision support systems for plantations: a new role for agronomists. The Planter, 73, 687-693.
} 
superficies plantées. Pobè, Bénin : Centre de Recherches Agricoles Plantes Pérennes (CRAPP).

Adjè A.I., 2004. Contribution à l'analyse du micro-climat, de la fertilité des sols et de la productivité des systèmes de culture à base du palmier à huile (Elaeis guineensis Jacq) au Sud-Bénin : cas des sites expérimentaux de Pobè et d'Obèkè-Ouèrè. Thèse de doctorat: Université Félix Houphouet Boigny, Abidjan-Cocody (Côte d'Ivoire).

Aho N. \& Kossou D.K., 1997. Précis d'agriculture tropicale. Cotonou, Bénin : Les Éditions du Flamboyant.

Aholoukpè N.S.H., 2013. Matière organique $d u$ sol et développement du palmier à huile sous différents modes de gestion des feuilles d'élagage. Cas des palmeraies villageoises du département du Plateau au Bénin. Thèse de doctorat : Université d'Abomey-Calavi (Bénin).

Aholoukpè $\mathrm{H}$. et al., 2013. Typologie des plantations villageoises de palmier à huile (Elaeis guineensis Jacq.) dans le département du Plateau au Bénin. Int. J. Biol. Chem. Sci., 7, 978-999.

Ahoyo N.R., 2008. Potentialités des filières anacarde et palmier à huile au Bénin. Rapport d'étude. Cotonou, Bénin : SNV Bénin.

Akpo E. et al., 2012. A participatory diagnostic study of oil palm (Elaeis guineesis) seed system in Benin. Wageningen J. Life Sci., 60-63, 15-27.

Amadji G.L. \& Aholoukpè H.N.S., 2008. Impact du niébé (Vigna unguiculata) et de la fumure minérale sur les propriétés chimiques de la terre de barre du Bénin. Étud. Gestion Sols, 15(3), 147-160.

Arrouays D. et al., éds, 2002. Contribution à la lutte contre l'effet de serre. Stocker du carbone dans les sols agricoles de France? Expertise Scientifique Collective. Synthèse du rapport. INRA.

Azontondé H.A., 1991. Propriétés physiques et hydrauliques des sols au Bénin. IAHS Publ., 199, 253-256.

Barcelos E., 1998. Étude de la diversité génétique du genre Elaeis (E. oleifera Kunth. Cortès et E. guineensis Jacq.) par marqueur moléculaires (RFLP et AFLP). Thèse de doctorat : École Nationale Supérieure Agronomique de Montpellier (France).

Barthes B. et al., 2004. Effect of a legume cover crop (Mucuna pruriens var. utilis) on soil carbon in an Ultisol under maize cultivation in southern Benin. Soil Use Manage., 20, 231-239.

Barthès B.G. et al., 2006. Determination of total carbon and nitrogen content in a range of tropical soils using near infrared spectroscopy: influence of replication and sample grinding and drying. J. Near Infrared Spectrosc., 14, 341-348.

Bernoux M. et al., 2011. Le programme GESSOL 2 : impact des pratiques agricoles sur les matières organiques et les fonctions des sols. Étud. Gestion Sols, 18(3), 137-145.

Brunet D., Barthès B., Chotte J.-L. \& Feller C., 2007. Determination of carbon and nitrogen contents in Alfisols, Oxisols and Ultisols from Africa and Brazil using NIRS analysis: effects of sample grinding and set heterogeneity. Geoderma, 139(1-2), 106-117.

Carlson K.M. et al., 2013. Carbon emissions from forest conversion by Kalimantan oil palm plantations. Nature Climate Change, 3, 283-287.

Chang C.-W., Laird D.A., Mausbach M.J. \& Hurburgh C.R. Jr., 2001. Near-infrared reflectance spectroscopyprincipal components regression analyses of soil properties. Soil Sci. Soc. Am. J., 65, 480-490.

Corley R.H.V. \& Tinker P.B., eds, 2003. The oil palm. $4^{\text {th }}$ ed. Oxford, UK: Blackwell Science.

Dassou O.S., 2011. Impact du choix de la méthode d'échantillonnage racinaire dans la détermination des paramètres de développement racinaire chez le palmier à huile (Elaeis guineensis Jacq.) au Bénin. Mémoire d'ingénieur agronome: Université d'Abomey-Calavi (Bénin).

Djegui N., 1992. Matière organique et azote dans les sols cultivés sur terres de barre (Bénin). Thèse de doctorat: École Nationale Supérieure d'Agronomie de Toulouse (France).

Djegui N., 1995. Les stocks organiques dans les sols cultivés sous palmeraie et cultures vivrières dans le sud du Bénin. In : Actes du séminaire, Fertilité du milieu et stratégies paysannes sous les tropiques humides, 13-17 novembre 1995, CIRAD, Montpellier, France, 189-193.

Djègui N., de Boissezon P. \& Gavinelli E., 1992. Statut organique d'un sol ferrallitique du Sud-Bénin sous forêt et différents systèmes de cultures. Cah. Orstom Sér. Pédol., 17(1), 5-22.

Dubos B., Alarcon H.W., Lopez J.E. \& Ollivier J., 2011. Potassium uptake and storage in oil palm organs: the role of chlorine and the influence of soil characteristics in the Magdalena valley, Colombia. Nutr. Cycl. Agroecosyst., 89, 219-227.

Ellert B.H. \& Bettany J.R., 1995. Calculation of organic matter and nutrients stored in soils under contrasting management regimes. Can. J. Sci., 75, 529-538.

Ellert B.H., Janzen H.H. \& Entz T., 2002. Assessment of a method to measure temporal change in soil carbon storage. Soil Sci. Soc. Am. J., 66, 1687-1695.

Frazão L.A., Paustian K., Pellegrino Cerri C.E. \& Cerri C.C., 2013. Soil carbon stocks and changes after oil palm introduction in the Brazilian Amazon. Global Change Biol. Bioenergy, 5, 384-390.

Frazão L.A.,Paustian K., Pellegrino Cerri C.E.\& Cerri C.C., 2014. Soil carbon stocks under oil palm plantations in Bahia State, Brazil. Biomass Bioenergy, 62, 1-7.

Goh K.J. \& Härdter R., 2003. General oil palm nutrition. In: Fairhurst T.H. \& Härdter R., eds. Oil palm management for large and sustainable yields. Singapore: Potash \& Phosphate Institute (PPI), Potash \& Phosphate Institute of Canada (PPIC) and International Potash Institute (IPI, Basel), 191-230.

Khalid H., Zin Z.Z. \& Anderson J.M., 1999. Quantification of oil palm biomass and nutrient value in a mature 
plantation. 1. Above-ground biomass. J. Oil Palm Res., 11(1), 23-32.

Khalid H., Zin Z.Z. \& Anderson J.M., 2000a. Soil nutrient dynamics and palm growth performance in relation to residue management pratices following replanting of oil palm plantation. J. Oil Palm Res., 12(1), 25-45.

Khalid H.,Zin Z.Z.\&Anderson J.M.,2000b.Decomposition processes and nutrients release patterns of oil palm residues. J. Oil Palm Res., 12(1), 46-63.

Lardy Chapuis L., Brossard M., Assad Lopes M.L. \& Laurent J.Y., 2002. Carbon and phosphorus stocks of clayey Ferralsols in Cerrado native and agroecosystems, Brazil. Agric. Ecosyst. Environ., 92, 147-158.

Law M.C. et al., 2009. Spatial variability of soil organic carbon in oil palm: a comparison between young and mature stands. Int. J. Agric. Res., 4(12), 402-417.

Littell R.C. et al., 2006. SAS for mixed models. $2^{\text {nd }}$ ed. Cary, NC, USA: SAS Institute Inc.

Nelson N.P. \& Su N., 2010. Soil pH buffering capacity: a descriptive function and its application to some acidic tropical soils. Aust. J. Soil Res., 48, 201-207.

Nelson N.P. et al., 2011. Soil acidification under oil palm: rates and effects on yield. Better Crops, 95(4), 22-25.

Nodichao L., 2008. Biodiversité racinaire, absorption potassique et résistance à la sécheresse chez le palmier à huile au Bénin. Thèse de doctorat: Université Félix Houphouet Boigny, Abidjan-Cocody (Côte d'Ivoire).

Nodichao L., Aké S. \& Jourdan C., 2008. Développement du système racinaire chez le palmier à huile selon l'origine génétique et le régime hydropotassique du sol. Agron. Afr., 20(3), 277-289.

Nouy B., Baudouin L., Djégui N. \& Omoré A., 1999. Le palmier à huile en conditions hydriques limitantes. Plant. Rech. Dev., 6(1), 31-45.

Pansu M.\& Gautheyrou J., 2006. Handbook of soil analysis. Mineralogical, organic and inorganic methods. Berlin; Heidelberg, Germany: Springer-Verlag.

Paramananthan, 2003. Land selection for oil palm. In: Fairhurst T. \& Härdter R., eds. Oil palm management for large and sustainable yields. Singapore: Potash \& Phosphate Institute (PPI), Potash \& Phosphate Institute of Canada (PPIC) and International Potash Institute (IPI, Basel), 27-57.

Reinhardt G., Retteinmaier N. \& von Falkenstein E., 2007. Conclusive evaluation of studies. Assessing the environmental impact of the use of palm oil as a bioenergy carrier. Final Report. Heidelberg, Germany: Institut für Energie- und Umweltforschung (IFEU).

SAS Institute Inc, 2011. SAS/STATß 9.3 User's Guide. Cary, NC, USA: SAS Institute Inc.

Volkoff B. \& Willaime P., 1976. Carte pédologique de reconnaissance de la République Populaire du Bénin. Feuille de Porto-Novo. Notice explicative $n^{\circ} 66(1)$. Paris : ORSTOM, 39, carte h.t.

(44 réf.)

Annexe 1. Paramètres physico-chimiques du sol sous RT (andain) et RN (interligne) des palmeraies adultes - Soil chemical parameters under TR (pruned fronds pile) and NR (interrow) of the adult oil palm plantations.

\begin{tabular}{|c|c|c|c|c|c|c|c|c|c|c|}
\hline \multirow[t]{2}{*}{ Zone } & \multirow{2}{*}{$\begin{array}{l}\text { Profondeur } \\
(\mathrm{cm})\end{array}$} & \multicolumn{2}{|l|}{$\mathbf{g} \cdot \mathbf{k g}^{-1}$} & \multirow[b]{2}{*}{$\mathrm{C} / \mathbf{N}$} & \multirow[t]{2}{*}{$\mathrm{pH} \mathbf{H}_{2} \mathbf{0}$} & \multicolumn{5}{|c|}{ meq.100 $\mathrm{g}^{-1}$} \\
\hline & & $\mathbf{C}$ & $\mathbf{N}$ & & & $\mathbf{C a}$ & Mg & $\mathbf{K}$ & $\mathbf{S}$ & CEC \\
\hline \multirow{5}{*}{$\begin{array}{l}\text { RT } \\
\text { (Andain) }\end{array}$} & $0-5$ & $31,28^{\mathrm{a}, \alpha}$ & $2,07^{\mathrm{a}, \alpha}$ & $15,22^{\mathrm{a}, \alpha}$ & $6,2^{\mathrm{a}, \alpha}$ & $7,32^{\mathrm{a}, \alpha}$ & $3,42^{\mathrm{a}, \alpha}$ & $0,17^{\mathrm{a}, \alpha}$ & $10,90^{\mathrm{a}, \alpha}$ & $9,00^{\mathrm{a}, \alpha}$ \\
\hline & $5-10$ & $19,29^{b, \alpha}$ & $1,26^{\mathrm{b}, \alpha}$ & $15,49^{\mathrm{a}, \alpha}$ & $6,0^{\mathrm{b}, \alpha}$ & $4,42^{b, \alpha}$ & $1,70^{\mathrm{b}, \alpha}$ & $0,09^{b, \alpha}$ & $6,21^{b, \alpha}$ & $5,85^{\mathrm{b}, \mathrm{a}}$ \\
\hline & $10-20$ & $10,12^{\mathrm{c}, \alpha}$ & $0,69^{\mathrm{c}, \alpha}$ & $14,62^{\mathrm{a}, \alpha}$ & $5,9^{\mathrm{b}, \alpha}$ & $2,84^{\mathrm{c}, \alpha}$ & $0,98^{\mathrm{c}, \alpha}$ & $0,05^{\mathrm{c}, \alpha}$ & $3,87^{\mathrm{c}, \alpha}$ & $4,05^{\mathrm{c}, \alpha}$ \\
\hline & $20-30$ & $4,98^{\mathrm{d}, \alpha}$ & $0,43^{\mathrm{d}, \alpha}$ & $11,49^{\mathrm{b}, \alpha}$ & $5,8^{\mathrm{b}, \alpha}$ & $2,01^{\mathrm{d}, \alpha}$ & $0,74^{\mathrm{c}, \alpha}$ & $0,03^{\mathrm{d}, \alpha}$ & $2,78^{\mathrm{d}, \alpha}$ & $3,40^{\mathrm{c}, \alpha}$ \\
\hline & $30-50$ & $3,55^{\mathrm{d}, \alpha}$ & $0,36^{\mathrm{d}, \alpha}$ & $9,65^{\mathrm{c}, \alpha}$ & $5,7^{\mathrm{b}, \alpha}$ & $2,03^{\mathrm{d}, \alpha}$ & $0,76^{\mathrm{c}, \alpha}$ & $0,02^{\mathrm{d}, \alpha}$ & $2,82^{\mathrm{d}, \alpha}$ & $3,80^{\mathrm{c}, \beta}$ \\
\hline \multirow{5}{*}{$\begin{array}{l}\text { RN } \\
\text { (Interligne) }\end{array}$} & $0-5$ & $12,08^{a, \beta}$ & $0,84^{\mathrm{a}, \beta}$ & $14,48^{\mathrm{a}, \alpha}$ & $5,5^{\mathrm{a}, \beta}$ & $2,68^{\mathrm{a}, \beta}$ & $0,87^{\mathrm{a}, \beta}$ & $0,06^{\mathrm{a}, \beta}$ & $3,62^{a, \beta}$ & $4,71^{\mathrm{a}, \beta}$ \\
\hline & $5-10$ & $9,04^{\mathrm{b}, \beta}$ & $0,64^{\mathrm{b}, \beta}$ & $13,92^{\mathrm{a}, \beta}$ & $5,6^{\mathrm{a}, \beta}$ & $2,40^{\mathrm{b}, \beta}$ & $0,70^{\mathrm{b}, \beta}$ & $0,04^{\mathrm{b}, \beta}$ & $3,14^{\mathrm{b}, \beta}$ & $3,81^{\mathrm{b}, \beta}$ \\
\hline & $10-20$ & $6,19^{c, \beta}$ & $0,49^{\mathrm{c}, \beta}$ & $12,45^{\mathrm{b}, \beta}$ & $5,7^{\mathrm{a}, \beta}$ & $2,20^{\mathrm{b}, \beta}$ & $0,64^{\mathrm{b}, \beta}$ & $0,03^{\mathrm{c}, \beta}$ & $2,86^{\mathrm{b}, \beta}$ & $3,50^{\mathrm{c}, \beta}$ \\
\hline & $20-30$ & $3,68^{\mathrm{d}, \alpha}$ & $0,36^{\mathrm{d}, \alpha}$ & $9,89^{\mathrm{c}, \beta}$ & $5,7^{\mathrm{a}, \alpha}$ & $1,84^{\mathrm{c}, \alpha}$ & $0,68^{\mathrm{b}, \alpha}$ & $0,02^{\mathrm{d}, \alpha}$ & $2,54^{\mathrm{c}, \alpha}$ & $3,35^{\mathrm{c}, \alpha}$ \\
\hline & $30-50$ & $3,41^{\mathrm{d}, \alpha}$ & $0,37^{\mathrm{d}, \alpha}$ & $8,63^{\mathrm{c}, \beta}$ & $5,7^{\mathrm{a}, \alpha}$ & $2,22^{b, \alpha}$ & $0,86^{\mathrm{a}, \alpha}$ & $0,02^{\mathrm{c}, \mathrm{d}, \alpha}$ & $3,10^{\mathrm{b}, \alpha}$ & $4,38^{\mathrm{d}, \mathrm{a}}$ \\
\hline
\end{tabular}

$\mathrm{S}$ : somme cations - som of cations ; CEC : capacité d'échange cationique - cationic exchange capacity; les lettres en exposant de chaque valeur indiquent la comparaison des moyennes selon le test de Tukey - superscript letters indicate the comparison of the means by the Tukey test ; les lettres romaines comparent les moyennes des profondeurs dans le même mode de gestion des feuilles d'élagage, alors que les lettres grecques comparent les moyennes d'une même profondeur entre les deux modes - Roman letters compare the soil layers of the same mode of pruned fronds management, while the Greek letters compare the same soil layer between the two modes. 